\title{
Effect of Antipsychotic Withdrawal on Negative Symptoms in Schizophrenia
}

Del D. Miller, Pharm.D., M.D., Michael Flaum, M.D., Stephan Arndt, Ph.D., Frank Fleming, B.S., B.S.N., and Nancy C. Andreasen, M.D., Ph.D.

\begin{abstract}
Although it is generally accepted that antipsychotic treatment improves the negative symptoms of schizophrenia in the context of improvement of positive symptoms, exactly how and to what extent they effect "primary" negative symptoms remains controversial. Antipsychotic treatment may reduce only those negative symptoms secondary to positive or depressive symptoms, and may have minimal, if any effect, on negative symptoms that represent a primary psychopathological trait manifestation of schizophrenia. In an effort to further examine this issue, we prospectively assessed negative, positive, depressive, and extrapyramidal symptoms following the discontinuation of antipsychotic medication. Fifty-nine DSM III-R schizophrenic patients underwent a three-week drug wash as part of our neuroimaging protocols. We assessed psychopathological status and adverse effects utilizing various rating instruments (i.e., Scale for Assessment of Positive
\end{abstract}

Symptoms [SAPS], Scale for Assessment of Negative Symptoms [SANS], Hamilton Rating Scale for Depression, and Simpson-Angus Extrapyramidal) at baseline and weekly during this three-week period. Negative symptoms, as measured by the SANS, worsened significantly during the three-week drug wash. Positive symptoms showed a less consistent change with symptoms of disorganization worsening and with psychotic symptoms remaining the same. The changes in negative symptoms during the drug-free period were correlated with the changes in psychosis and disorganization, but not with changes in depression or extrapyramidal side effects. We were not able to substantiate if the worsening in negative symptoms was a direct result of the worsening of positive symptoms or if they were changing simultaneously, but independent of each other. [Neuropsychopharmacology 11:11-20, 1994]
KEY WORDS: Negative symptoms; Antipsychotic withdrawal

Schizophrenia is a complex disease characterized by a multiplicity of symptoms affecting aspects of human cognition, emotion, and behavior. In an attempt to bring coherence to the wide range of signs and symptoms

From the Mental Health Clinical Research Center, University of Iowa Hospitals and Clinics, Iowa City, IA.

Presented in part at the 31st Annual Meeting of the American College of Neuropsychopharmacology, December 18, 1992, San Juan, Puerto Rico.

Address reprint requests to: Del D. Miller, Pharm.D., M.D., University of lowa Hospitals and Clinics, Department of Psychiatry Administration, Rm 2887 JPP, 200 Hawkins Drive, Iowa City, IA 52242-1057.

Received December 10, 1992; revised February 2, 1994; accepted February 28, 1994. associated with schizophrenia, they have been divided into two major categories: positive symptoms and negative symptoms. Using this archetype, positive symptoms as a group represent a distortion or excess of normal function, and include hallucinations (disturbance of perception), delusions (disturbance of cognition), formal thought disorder (disturbance of language), and bizarre behavior (disturbance in behavioral control). On the other hand, negative symptoms represent a loss or diminution of normal function and are comprised of affective flattening (restricted experience of emotion and restricted expressiveness), avolition (loss of volition and drive), anhedonia (loss of interest), and alogia (loss of fluency of thought) (Jackson 1875).

Although the importance of negative symptoms were recognized by both Kraepelin (1919) and Bleuler 
(1950) as the most important symptoms of schizophrenia, they were virtually ignored in psychiatric nosology during the 1970s and mid-1980s because of the relative weight given to positive symptoms, especially frst-rank symptoms. The renewed interest in negative symptoms within the past decade is partly due to the work of Strauss and Carpender (1974), Crow (1980), and Andreasen (1982), who emphasized the ubiquitous nature of these symptoms, and the excess morbidity associated with them. With this increased interest in negative symptoms, research investigating neurobiological correlates, pathophysiology, and the treatment of negative symptoms has blossomed.

Prior to the 1980s, the pharmacological treatment of schizophrenia focused almost exclusively on the reduction of florid symptoms such as hallucinations, delusions, bizarre behavior, and formal thought disorder with little attention to negative symptoms. Research examining the efficacy of antipsychotics in treating negative symptoms was stimulated by Crow's (1980) intriguing proposal that the negative symptoms of schizophrenia are generally not responsive to pharmacological treatment. This inference was based primarily on a study by Johnstone, et al. (1978), who demonstrated that cis-flupenthixol, an effective dopamine, D2, receptor antagonist in the thioxanthine class, was no more effective in treating negative symptoms than its dopaminergically inactiveisomer. At least three other groups have reported findings suggesting that negative symptoms are not effectively treated by typical antipsychotics (Clark et al. 1963; Serafetinides et al. 1972; Angrist et al. 1980). In contrast, there have been reports revealing that negative symptoms can be partially improved in a subgroup of schizophrenics treated with conventional antipsychotics (Goldberg 1965; Breier et al. 1987; Angst et al. 1989; Coryell et al. 1990; Tandon et al. 1990; Meltzer et al. 1991), and a number of studies indicating that the atypical antipsychotics like clozapine (Kane et al. 1988; Miller et al. 1994) and risperidone (Castelão et al. 1989; Bressa et al. 1991; Chouinard et al. 1993) may be even more effective in ameliorating negative symptoms. There is now a general consensus that antipsychotics reduce negative symptoms during the successful treatment of positive symptoms, but that they improve at a slower rate and not as thoroughly as positive symptoms improve (Meltzer et al. 1986). What remains controversial is if negative symptoms that represent a primary psychopathological trait manifestation of schizophrenia are effectively treated by antipsychotic medications.

One of the most difficult problems in the study of negative symptoms in schizophrenia involves the recognition that objectively assessable negative symptoms may occur as a consequence of a variety of factors. They can be "primary," related to the underlying pathophysiology of schizophrenia, or they can be "secondary" to several distinct pathogenic mechanisms. The most commonly implicated mechanisms responsible for "secondary" negative symptoms include: (1) positive symptoms (e.g., social avoidance secondary to paranoia); (2) neuroleptic side effects (e.g., akinesia); (3) depression (which is common in schizophrenia, particularly during the residual phase) (Sirus 1991); and (4) environmental understimulation resulting from chronic institutionalization. Attempting to assess changes in negative symptoms in patients whose pharmacological treatment is being manipulated, and in particular to determine the effect of a specific pharmacological treatment on negative symptoms, is therefore very complex. Pharmacological treatment may have different effects on "primary" and/or "secondary" factors that are subsequently reflected as changes in ratings of negative symptoms.

Studies that have reported an improvement in negative symptoms with antipsychotic treatment, generally have discovered that the change in negative symptoms occurred in the context of an improvement in positive symptoms (Goldberg 1985; Breier et al. 1987; Angst et al. 1989; Coryell et al. 1990; Tandon et al. 1990; Meltzer et al. 1991). However, the investigators were unable to ascertain if this was a direct cause-and-effect relationship or an independent, simultaneous improvement. Others have proposed that reduction in extrapyramidal symptoms (EPS) and/or depression can also mimic improvement in negative symptoms and need to be accounted for (Carpenter et al. 1985a). Although several of the studies analyzed the correlation between the changes in negative symptoms and the changes in positive symptoms (Breier et al. 1987; Tandon et al. 1990), none examined the degree to which improvement in negative symptoms was correlated with a reduction in depression or EPS.

Another approach towards investigating the effect that antipsychotics have on negative symptoms has been to monitor negative symptoms during antipsychotic withdrawal (Naber et al. 1985; Breier et al. 1987). These studies assume that the change in negative symptoms associated with antipsychotic withdrawal is opposite the effect on negative symptoms that occurs with antipsychotic treatment (i.e., if negative symptoms worsen following antipsychotic discontinuation, it is assumed that the antipsychotics had previously been effective in reducing negative symptoms). Naber and colleagues (1985) studied a group of 36 chronic, schizophrenic patients who were rated clinically while receiving neuroleptic therapy and who were rated during 12 days of antipsychotic withdrawal. They reported that discontinuation of long-term neuroleptic treatment was associated with improvement in the BPRS anergic scores and the deterioration of BPRS thought disorder scores. In contrast, Breier and associates (1987) observed that there was an exacerbation in both negative and 
positive symptoms in a group of 19 young schizophrenics who were withdrawn from antipsychotics for four weeks. There was no correlation between the change in the BPRS negative symptom rating and the change in BPRS positive symptoms cluster ratings. Neither study used standardized assessment instruments specifically design to rate negative symptoms nor thoroughly investigated if interactions of changes in other symptoms (i.e., depression and/or EPS) were mimicking a change in negative symptoms.

In a further attempt to evaluate the effect of antipsychotics on negative symptoms, we examined the changes in negative and positive symptoms, ratings of depression, and EPS in a group of 59 chronic schizophrenics who were withdrawn from antipsychotic medications for 3 weeks. We also explored the interrelationship between the changes in the various symptoms to determine if the change in negative symptoms was secondary to changes in other symptoms. We hypothesized that negative symptoms would increase as positive and depressive symptoms increased, and that the changes would be correlated. We further hypothesized that this worsening of negative symptoms would occur even though there would be a simultaneous reduction in EPS.

\section{METHODS}

\section{Subjects}

We evaluated a sample of fifty-nine consecutive patients who gave written, informed consent, before they underwent a three-week antipsychotic medication washout as part of a protocol for the University of Iowa Mental Health Clinical Research Center. All patients met DSM III-R (1987) criteria for schizophrenia and had been receiving an oral antipsychotic prior to the study. Individuals who had received depot antipsychotic within the previous six months or had co-existing medical problems were excluded. There were 18 female and 41 male subjects in the sample with a mean age of $32.6 \pm$ 9.8 years. They had been ill for $13.2 \pm 4.5$ years and had $4.6 \pm 4.2$ previous hospitalizations. Forty-three of the 59 subjects were part of the data base used to demonstrate the usefulness of various statistical methods for analyzing repeated measures of data (Arndt et al. 1993).

\section{Procedures}

After an initial baseline assessment period of 3 to 5 days, patients' antipsychotic medications were tapered and discontinued over a 2 to 3 day period. They then remained off medication for 3 weeks. The wash was terminated if a patient requested that it be discontinued or if the patient became threatening or physically ag- gressive. Just prior to the drug discontinuation, 23 patients had been taking haloperidol (mean dose 26.9 $\mathrm{mg} /$ day; with a range of 5 to $85 \mathrm{mg} /$ day), 18 had been taking thiothixene (mean dose $37.4 \mathrm{mg} /$ day; with a range of 5 to $80 \mathrm{mg} /$ day), 7 had been taking fluphenazine (mean dose $24.6 \mathrm{mg} /$ day; with a range of 2 to 60 $\mathrm{mg} /$ day), 3 had been taking trifluoperazine (mean dose $23.0 \mathrm{mg} /$ day; with a range of 4 to $50 \mathrm{mg} /$ day), $2 \mathrm{had}$ been taking chlorpromazine (mean dose $200.0 \mathrm{mg}$ /day), $2 \mathrm{had}$ been taking molindone (mean dose $50.0 \mathrm{mg} /$ day; with a range of 25 to $75 \mathrm{mg} /$ day), 1 had been taking loxapine (dose $100 \mathrm{mg} /$ day), and 3 had been taking combinations of two different antipsychotics (thioridazine and fluphenazine, trifluoperazine and chlorpromazine, and trifluoperazine and thioridazine).

\section{Clinical Assessment}

Patients were assessed clinically by means of the CASH (Andreasen 1987; Andreasen et al. 1992). This structured interview, developed at our center to assess patients with major psychoses, provides comprehensive details of sociodemographic data including educational history, work history, and social history. It also contains several standard scales used to rigorously assess psychopathology, such as the Scale for Assessment of Positive Symptoms (SAPS) (Andreasen 1984), Scale for Assessment of Negative Symptoms (SANS) (Andreasen 1982; Andreasen 1983), the Global Assessment Scale (GAS) (Endicott et al. 1976), the Mini-Mental State Examination (MMSE) (Folstein et al. 1975), and the Premorbid Asocial Adjustment Scale (Gittelman-Klein and Klein 1969). Assessments were determined, based on direct observation of the patient, the self-report during the interview, the interviews with informants, the reports by nursing personnel, and the reports from referring physicians.

Research nurses completed ratings of psychopathology and side effects upon inclusion in the study, and then weekly during the drug washout. Assessment instruments included the SANS (Andreasen 1982; Andreasen 1983), SAPS (Andreasen 1984), Hamilton Rating Scale for Depression (HAM-D) (Hamilton 1960), Simpson-Angus rating scale for Extrapyramidal Symptoms (SA-EPS) (Simpson and Angus 1970), and the Abnormal Involuntary Movement Scale (AIMS). Raters participate in an ongoing training program and must maintain a minimum level of competence on the rating instruments used. At our center, the interrater reliability of the individual SAPS and SANS global items ranges from 0.62 to 0.93 (Andreasen et al. 1992).

Ratings from the SAPS and SANS were divided into three symptom dimensions (negative symptom dimension, psychoticism dimension, and disorganization dimension) described in previous factor-analytic studies (Bilder et al. 1985; Andreasen and Grove 1986; 
Table 1. Weekly Clinical Ratings of 51 Schizophrenics Who Completed a 3-Week Antipsychotic Wash

\begin{tabular}{lccccc}
\hline & Baseline & $\begin{array}{c}\text { Week 1 } \\
\text { Wash }\end{array}$ & $\begin{array}{c}\text { Week 2 } \\
\text { Wash }\end{array}$ & $\begin{array}{c}\text { Week 3 } \\
\text { Wash }\end{array}$ & $\begin{array}{c}\text { CMH- } \\
\text { Monotonic } \\
\boldsymbol{p} \text { Value }\end{array}$ \\
\hline $\begin{array}{l}\text { Negative symptom } \\
\text { dimension }\end{array}$ & $11.1 \pm 3.9$ & $11.3 \pm 3.9$ & $13.1 \pm 4.5$ & $13.1 \pm 4.4$ & 0.001 \\
$\begin{array}{l}\text { Disorganization } \\
\text { dimension }\end{array}$ & $3.2 \pm 2.9$ & $4.1 \pm 3.1$ & $5.0 \pm 3.6$ & $5.3 \pm 3.5$ & 0.0001 \\
$\begin{array}{l}\text { Psychoticism } \\
\text { dimension }\end{array}$ & $5.5 \pm 2.8$ & $5.0 \pm 2.7$ & $5.5 \pm 2.5$ & $5.1 \pm 3.2$ & 0.98 \\
$\begin{array}{l}\text { Simpson } \\
\quad \text { Angus }\end{array}$ & $2.7 \pm 3.3$ & $2.4 \pm 3.7$ & $2.0 \pm 2.9$ & $1.5 \pm 2.1$ & 0.027 \\
$\begin{array}{l}\text { HAM-D (mood) } \\
\text { AIMS }\end{array}$ & $1.7 \pm 2.3$ & $1.9 \pm 2.4$ & $2.4 \pm 2.5$ & $2.4 \pm 2.6$ & 0.40 \\
GAS & $2.3 \pm 3.3$ & $2.8 \pm 4.3$ & $3.3 \pm 4.8$ & $4.0 \pm 5.3$ & 0.19 \\
\hline$\quad$ df $=1$. & $34.6 \pm 8.5$ & $30.8 \pm 9.3$ & $28.5 \pm 11.5$ & $28.1 \pm 10.9$ & 0.0001 \\
\hline
\end{tabular}

Liddle 1987; Gur et al. 1991; Arndt et al. 1991; Miller et al. 1993). The Psychoticism Dimension included the global ratings of hallucinations and delusions; the Disorganization Dimension was comprised of the global ratings of bizarre behavior, formal thought disorder and inappropriate affect, and Negative Symptom Dimension was comprised of the global ratings of affective flattening, alogia, avolition-apathy, and anhedoniaasociality from the SANS (Amdt et al. 1991; Miller et al. 1993).

Using a principal component analysis, Goldman et al. (1992) reported that there was a large overlap between many items on the HAM-D and SANS negative symptoms in schizophrenia and suggested that the HAM-D items of depressed mood, guilt, and suicidal ideation represent "core" depressive symptoms in schizophrenic patients. Based on these findings, we used a sum of the HAM-D items of depressed mood, guilt, and suicidal ideation as an independent measure of depression, which is referred to as HAM-D (mood).

\section{Data Analysis}

The mean baseline and the weekly ratings of psychopathology and side effects were compared to determine if there was a change over the three-week drug wash. Prior to analyzing the seven dependent variables with a standard repeated measures ANOVA (i.e., randomized block design), checks for the assumed condition of sphericity (Ekstrom et al. 1990) were made with the likelihood ratio test (Mauchly 1940; Rogan et al. 1984). In six of the seven scales, the sphericity condition was untenable $(p<0.05)$. Because only one of the seven seemed to satisfy the condition, we used the multivariate analog of repeated measures, ANOVA, to obtain tests for overall mean differences.

As many of the variables were distinctively nonnormally distributed, Cochran-Mantel-Haenszel (CMH) analyses of ranks were performed on each variable in parallel with the MANOVAs (Agresti 1990). An inspection of the means over the four evaluations indicated an almost steady increase or decline in scores, therefore, the $\mathrm{CMH}$ test for monotonic correlation was applied. We have previously proven that the $\mathrm{CMH}$ is a particularly powerful method to analyze repeated measures as it places fewer restrictions on the data, and is more sensitive to patterns of change (Amdt et al. 1993).

As discussed previously, a critical issue in the consideration of the effect of antipsychotic medication on negative symptoms is if any of this effect is independent of the effect on positive symptoms, the effect of EPS, and/or the effect on depression. We utilized several methods in an attempt to disentangle these possible interrelationships. First, changes between baseline and week three were calculated for each patient on each scale. Spearman correlations between changes in negative symptoms and changes in positive symptoms (disorganization dimension and psychoticism dimension), changes in extrapyramidal side effects measured by the SA-EPS, and changes in depression rated on the HAM$D$ (mood) were performed to determine if the negative symptoms were changing independently of these items that are known to engender "secondary" forms of negative symptoms. Next, we used a multiple regression model, with the change in ratings of negative symptoms as the dependent measure in a model that included the change in psychotic symptoms, the change in disorganization, the change in EPS, and the change in depressive symptoms.

\section{RESULTS}

Fifty-nine patients met the inclusion criteria, provided informed consent, and entered the drug wash. Fiftyone $(86.4 \%)$ completed the three-week wash. Of the 
Table 2. Correlations between Changes in Symptom Rating from Baseline to Week 3 During an Antipsychotic Wash $(n=51)$

\begin{tabular}{|c|c|c|c|c|c|}
\hline & $\begin{array}{c}\text { Negative } \\
\text { Symptom } \\
\text { Factor }\end{array}$ & $\begin{array}{c}\text { Disorganization } \\
\text { Factor } \\
\end{array}$ & $\begin{array}{c}\text { Psychoticism } \\
\text { Factor }\end{array}$ & $\begin{array}{c}\text { Simpson } \\
\text { Angus }\end{array}$ & $\begin{array}{l}\text { HAM-D } \\
\text { (mood) }\end{array}$ \\
\hline $\begin{array}{l}\text { Negative } \\
\text { symptom } \\
\text { factor }\end{array}$ & 1.00 & & & & \\
\hline $\begin{array}{l}\text { Disorganization } \\
\text { factor } \\
\text { Psychoticism }\end{array}$ & $0.38^{\star}$ & 1.00 & & & \\
\hline factor & $0.41^{\star}$ & $0.44^{\star}$ & 1.00 & & \\
\hline Simpson Angus & 0.14 & 0.07 & -0.08 & 1.00 & \\
\hline HAM-D (mood) & 0.21 & -0.18 & 0.08 & -0.3 & 1.00 \\
\hline
\end{tabular}

eight patients who did not complete the wash, 3 became threatening or physically aggressive and 5 requested that the wash be terminated. There were no differences in the age, gender, duration of illness, or number of hospitalizations between those who completed the wash and those who did not. There were also no significant differences in any rating of psychopathology at baseline, week 1 , or a change from baseline to week 1 between those who completed the three week drug-free period and those who did not.

The weekly means and standard deviations of the ratings of psychopathology and side effects appear in Table 1. Three of the seven ratings showed significant changes using both methods of analysis (i.e., MANOVA and $\mathrm{CMH}$ ). The Simpson-Angus scale for EPS was close to statistical significance using the MANOVA, and was significant using the $\mathrm{CMH}$ test for monotonic correlation. This consistency indicates that the findings are not an artifact of the particular analysis technique nor attributable to violations of statistical assumptions. For simplicity, only the results of the $\mathrm{CMH}$ monotonic analysis are shown.

During the three-week drug washout, we noted a significant worsening of the mean ratings of the negative symptom dimension. Although not shown in tabular form, the individual ratings of the four global negative symptoms (affective flattening, alogia, avolition-apathy, and anhedonia-asociality) all worsened significantly over the three-week period. The changes in the positive symptoms were not as straightforward, with the mean ratings of the disorganization symptom dimension worsening significantly, and the mean ratings of the psychotic symptom dimension not showing significant change. There was also a significant worsening of the mean ratings of GAS. In comparison, the mean ratings of EPS decreased significantly. There were no significant mean changes in ratings of depression or tardive dyskinesia during the three-week period.

Correlations between change scores are shown in
Table 2. As there was a significant worsening in mean ratings of the negative and disorganization symptom dimensions, we were particularly curious if the worsening in negative symptoms was correlated with the worsening in disorganization symptoms. We were also interested if individual changes in ratings of psychotic symptoms or depression were correlated with individual changes in negative symptoms, as these could also be involved in the change in negative symptoms. The changes in the negative symptom dimension was correlated with changes in both disorganization and psychosis $\left(r^{2}=0.14, p=.02 ; r^{2}=.18, p=.03\right)$. The changes in EPS and HAM-D-(mood) were not correlated with the quantified changes in negative symptoms or with the changes in symptoms of disorganization and psychosis.

As changes in disorganization, psychoticism, antipsychotic side effects, and depression could each be contributing to the observed change in negative symptoms, they were entered into a multiple regression model. We examined if change in psychoticism, in disorganization, in extrapyramidal symptoms, and/or in depression could predict the change in negative symptom ratings while controlling for the others and if the overall change in negative symptoms was significantly contributed to by a cumulative effect of the various items. The cumulative effect of the model accurately predicted the change in negative symptoms rating $(\mathrm{F}=2.94 ; d f=4 ; p=.039)$. However, none of the individual changes alone, accurately predicted the change in negative symptoms when controlling for the changes in the other symptoms (disorganization $\mathrm{F}=2.53, d f=$ $1, p=.123$; psychoticism $\mathrm{F}=2.35, d f=1, p=.137$; Simpson-Angus EPS $\mathrm{F}=.81, d f=1 ; p=.377$; depression $\mathrm{F}=2.21, d f=1 ; p=.148)$. We attempted to determine which symptoms were contributing to this finding. Because the change in psychotic symptoms and the change in disorganization were individually correlated with the change in negative symptoms, these 
two items were entered into a multiregression model to assess if they could accurately predict change in negative symptoms. These two items alone, robustly predicted the change in negative symptoms (F 4.21; $d f=$ $2 ; p=.025)$. The inclusion of change in extrapyramidal symptoms $(F=.78 ; d f=1 ; p=.383)$ and the change in depressive symptoms $(\mathrm{F}=2.24 ; d f=1 ; p=.146)$ did not significantly add to this model.

\section{DISCUSSION}

Antipsychotics have been used extensively in the treatment of individuals with schizophrenia for almost four decades, and yet if they benefit or worsen "primary" negative symptoms remains unclear. This largely reflects the difficulties inherent in the assessment of negative symptoms. Antipsychotics may well benefit those negative symptoms resulting from one cause, and exacerbate those of another. The most obvious example is antipsychotics decreasing both positive symptoms and dysphoria, leading to a decrease in negative symptoms, but also, causing drug-induced akinesia, manifesting objectively as affective flattening. Thus, the assessment of the efficacy of these medications on negative symptoms is complex. Whereas there is consensus that it is essential to attempt to disentangle "primary" versus "secondary" negative symptoms, there has been much debate about the best method of doing so.

There are at least two strategies in approaching this problem. One, that has been proposed by Carpenter and colleagues, involves a determination at the level of the rater as to whether negative symptoms are primary or secondary. They have developed specific criteria and a rating instrument, and using these along with adequate training, have demonstrated good levels of reliability, and several potential validators (Buchanan et al. 1989; Buchanan et al. 1990; Carpenter et al. 1985b; Kirkpatrick et al. 1989b).

However, concerns about the reliability and validity of this approach persist. Negative symptoms as a whole are difficult to rate. In our center, we have observed that great care must be taken to maintain adequate levels of reliability and calibration among raters, even in the context of an ongoing training program, and at a site where the assessment of negative symptoms has been a focus for many years. Concerns about reliability are supported by unpublished data from the psychotic disorders field trial for DSM-IV that examined the issue of distinguishing between "primary" and "secondary" negative symptoms without specific training or criteria. The question was added to the instrument used in the DSM-IV field trial for psychotic disorders that contained a SANS-based component. The mean inter-rater and test-retest reliability of making the primary versus secondary distinction across four negative symptoms was 0.54 and 0.35 respectively (Flaum et al. 1992; unpublished data).

The alternate approach involves ratings of negative symptoms based on objective measures, with concomitant assessment of the putative confounding factors (i.e., positive symptoms, depression, akinesia) and then determining if changes in negative symptoms are related to changes in these potential causes of secondary negative symptoms. If the changes are interrelated, the assumption is that the change in the negative symptoms is likely due to change in "secondary" negative symptoms, although this does not eliminate the possibility that they may be changing at similar rates, but independent of each other. Because this method obviates the need for potentially unreliable judgments regarding the source of a particular symptom, it is more cumbersome. Using such an approach, we discovered that both the means of the negative symptoms and the disorganization worsened significantly whereas the mean of the psychotic symptoms, depressive symptoms, and EPS did not change during the three weeks following the discontinuation of antipsychotic medication. The individual changes in psychosis and disorganization were positively correlated with the individual change in negative symptoms, whereas, the individual changes in depression and EPS were not. Likewise, the changes in psychosis and the changes in disorganization accurately predicted the change in negative symptoms in a multiple regression analysis, while the change in depression and EPS did not add significantly to this model.

There have been numerous studies examining response to antipsychotic discontinuation with the majority using "relapse" as their primary outcome measure (Prien et al. 1972; Andrews et al. 1976; Hogarty et al. 1976; Marder et al. 1979; Brown and Laughren 1981; Weinberger et al. 1981; Zander et al. 1981; Heinricks and Carpenter 1985; Perényi et al. 1985; Dencker et al. 1986; Lieberman et al. 1987; Glazer et al. 1989; Kirkpatrick et al. 1989a; van Kammen et al. 1989; Green et al. 1990; Davidson et al. 1991; Dixon et al. 1993). Investigators have utilized a variety of criteria for a definition of relapse, that tends to focus on positive symptomatology, and relatively few have reported mean changes in negative symptomatology. In these various studies, withdrawal of antipsychotic medication was reported to produce a heterogeneous response in psychopathology with relapse rates ranging from $25 \%$ to $60 \%$. Our finding of the mean ratings of negative symptoms worsening significantly following antipsychotic discontinuation is in agreement with the findings of Breier and co-workers (1987) who reported similar results, but differs from those of Dixon and colleagues (1993) who saw no change in negative symptoms, and from Naber and associates (1985) who discovered that negative symptoms improved after antipsychotics were 
discontinued. In the presentstudy, antipsychotics were discontinued for 3-weeks compared to 4-weeks in the Breier and Dixon studies and only 12 days in the Naber study. It is possible that negative symptoms may have worsened if the patients in the Naber study had remained off antipsychotics for a longer period of time. Other studies have noted variable responses of negative symptoms during antipsychotic discontinuation with some individuals showing improvement, some remaining the same, and others worsening (Hogarty et al. 1976; Dencker et al. 1986; Glazer et al. 1989; van Kammen et al. 1989). Although we discovered that the mean ratings of negative symptoms worsened significantly during the 3-week period, there was variability within the group with negative symptoms improving in 13 patients, not changing in 6 patients, and worsening in 32 patients.

In the present study, positive symptoms showed less consistent results with the mean symptom ratings of disorganization worsening, and the mean ratings of psychotic symptoms remaining unchanged. Utilizing the BPRS, Breier et al. (1987) and Naber et al. (1985) reported an exacerbation of positive symptoms, whereas, Dixon et al. (1993) found them not to change. The differences between our findings and the other studies is most likely related to the differences in the rating instruments as well as that they analyzed the data using two-dimensional models rather than the threedimensional model used here; therefore, if we combine psychotic and disorganization symptoms and define them as "positive" symptoms, we also demonstrate an overall worsening in the mean ratings of positive symptomatology. Even though mean ratings of disorganization and psychosis worsened and remained unchanged, respectively, there was variability of response among the patients. Eleven patients experienced improvement in disorganization, 5 showed no change, and 35 worsened; 21 patients showed an improvement in ratings of psychosis, 14 did not change, and 16 worsened. A possible explanation for the smaller increase in psychotic symptoms in the present study is the duration of the medication free period. The three-week period in the present study is relatively brief compared to the duration used in the other investigations (Prien et al. 1972; Andrews et al. 1976; Hogarty et al. 1976; Brown and Laughren 1981; Perényi et al. 1985; Dencker et al. 1986; Lieberman et al. 1987; van Kammen et al. 1989; Green et al. 1990; Davidson et al. 1991) and it is likely that we would have seen a greater worsening of psychosis if the drug-free period had been extended.

None of the studies analyzing the effect of antipsychotic discontinuation on negative symptoms have investigated the interrelationships between the changes in negative symptoms and the changes in positive symptoms, depression, or EPS. Utilizing the "core" depressive HAM-D items (depressed mood, guilt, and suicidal ideation) reported by Goldman (1992) as our measure of depression, we discovered no correlation between the change in the sum of the core items and the changes in negative symptoms. This suggests that the increase in negative symptoms was not related to the worsening of depression, however, when the total HAM-D score was used, there was a strong correlation between changes in HAM-D and changes in negative symptoms $\left(r^{2}=.36, p=.0003\right)$. In examining individual HAM-D items, it appeared that the increase in total HAM-D scores were largely contributed to by increases in the insomnia items as well as in items such as decreased work and interests, and psychomotor retardation. The HAM-D items of decreased work and interests, and psychomotor retardation appear to be a measure as much related to negative symptoms as to depression, particularly in schizophrenic populations. These findings confirm Goldman's conclusion that the HAM-D total score is a nonspecific measure of a variety of types of symptoms (depression, negative symptoms, agitation, and somatic distress) in schizophrenic patients. We agree that more specific and sensitive assessment techniques are needed to assess depression in schizophrenic patients.

EPS decreased significantly during the period off antipsychotic medication, and regarding negative symptoms, it would be expected that it would reduce them; therefore, it is not surprising that the observed change in EPS was not associated with a change in negative symptoms. Other forms of "secondary" negative symptoms that these data do not account for include environmental influences; for example, being in the hospital for three weeks may be considered demoralizing and amotivating, and may lead to behavior which is negative. Most investigators agree that demoralization and environmental deprivation may produce secondary negative symptoms, however, this is difficult to quantify. The supposition that environmental deprivation produces negative symptoms is speculatively based on literature relating chronicity to general effects of social and intellectual isolation. We did not have the ability to measure demoralization and environmental deprivation and were therefore, unable to ascertain if a change in them during the drug-free period may have been portrayed as a worsening of negative symptoms.

In summary, although these data lend some overall support to the idea that typical antipsychotics are efficacious in the treatment of negative symptoms, they do not clearly inform the underlying mechanisms. Negative symptoms increased significantly following discontinuation of antipsychotic medication, and this increase was associated with changes in psychosis and disorganization suggesting that at least a portion of this change was caused by a worsening of "secondary" negative symptoms; however, the changes in the various putative causes of secondary negative symptoms 
only accounted for $30 \%$ of the variance of the change in negative symptoms, inferring that other determinants, possibly involving a change in "primary" negative symptoms may also be occurring. Given the vast morbidity associated with negative symptoms, and the ubiquitous use of antipsychotics in the treatment of schizophrenia, investigators must continue to employ a variety of approaches in an effort to tease apart this important and complex relationship. Future research needs to focus on schizophrenic patients with prominent negative symptoms, who have minimal, if any, "secondary causes" of negative symptoms (i.e., positive symptoms, depression, or EPS), to determine if antipsychotics have a direct effect on "primary" negative symptoms.

\section{ACKNOWLEDGMENT}

This work was funded in part by NIMH Grant MH43271.

\section{REFERENCES}

Andrews P, Hall J, Snaith R (1976): A controlled trial of phenothiazine withdrawal in chronic schizophrenic patients. Br J Psychiatry 128:451-455

Agresti A (1990): Categorical data analysis. New York, John Wiley

American Psychiatric Association and Committee on Nomenclature and Statistics: Diagnostic and Statistical Manual of Mental Disorders, (1987): 3rd Edition-Revised (DMSIII-R), Washington, DC, American Psychiatric Press

Andreasen NC (1982): Negative symptoms in schizophrenia: Definition and reliability. Arch Gen Psychiatry 39:784-788

Andreasen NC (1983): The Scale for the Assessment of Negative Symptoms (SANS). Iowa City, Iowa; University of Iowa

Andreasen NC (1984): The Scale for the Assessment of Positive Symptoms (SAPS). Iowa City, Iowa: University of Iowa

Andreasen NC (1987): Comprehensive Assessment of Symptoms and History (CASH). Iowa City, Iowa: University of Iowa

Andreasen NC, Flaum M, Arndt S (1992): The Comprehensive Assessment of Symptoms and History (CASH): An instrument for assessing psychopathology and diagnosis. Arch Gen Psychiatry 49:615-623

Andreasen NC, Grove WM (1986): Evaluation of positive and negative symptoms in schizophrenia. Psychiatry and Psychobiology 1:108-121

Angrist B, Rotrosen J, Gershon S (1980): Differential effects of amphetamine and neuroleptics on negative versus positive symptoms in schizophrenia. Psychopharmacology 72:17-19

Angst J, Stassen HH, Woggon B (1989): Effect of neuroleptics on positive and negative symptoms and the deficit state. Psychopharmacology 99:S41-S46
Amdt S, Alliger RJ, Andreasen NC (1991): The distinction of positive and negative symptoms: the failure of a twodimensional model. Br J Psychiatry 158:317-322

Arndt S, Davis CS, Miller DD, Andreasen NC (1993): Effect of antipsychotic withdrawal on extrapyramidal symptoms: Statistical methods for analyzing single sample repeated measures data. Neuropsychopharmacology 8:67-75

Bilder RM, Mukherjee S, Rieder RO, Pandurangi A (1985): Symptomatic and neuropsychological components of defect states. Schizophr Bull 11:409-419

Bleuler E (1950): Dementia Praecox or the Group of Schizophrenias (translated by Zinkin J.). New York, International Universities Press

Breier A, Wolkowitz OM, Doran AR, Roy A, Boronow J, Hommer DW, Pickar D (1987): Neuroleptic responsivity of positive and negative symptoms in schizophrenia. Am J Psychiatry 144:1549-1555

Bressa GM, Bersani G, Meco G, Bouciqué E, Pozzi F (1991): One year follow-up study with risperidone in chronic schizophrenia. New Trends in Experimental and Clinical Psychiatry 7:169-177

Brown W, Laughren T (1981): Low serum prolactin and early relapse following neuroleptic withdrawal. Am J Psychiatry 138:237-239

Buchanan RW, Kirkpatrick B, Heinrichs DW, Carpenter WT (1990): Clinical correlates of deficit syndrome of schizophrenia. Am J Psychiatry 147:290-294

Buchanan RW, Kirkpatrick B, Tamminga CA (1989): Differential patterns of glucose metabolism in deficit and nondeficit schizophrenia. Biol Psychiatry 27(A):99A-100A

Carpenter W, Hanlon T, Heinrichs D, Summerfelt A, Kirkpatrick B, Levine J, Buchanan R (1990): Continuous versus targeted medication in schizophrenic outpatients: Outcome results. Am J Psychiatry 147:1138-1148

Carpenter WT, Heinrichs DW, Alphs LD. (1985a): Treatment of negative symptoms. Schizophr Bull 11:440-447

Carpenter WT, Heinrichs DW, Wagman AMI (1985b): Deficit and non-deficit forms of schizophrenia: The concept. Am J Psychiatry 145:578-583

Castelão JF, Ferreira L, Gelders YG, Heylen SLE (1989): The efficacy of the D2 and 5-HT2 antagonist risperidone (R64 766) in the treatment of chronic psychosis: An open dosefinding study. Schizophr Res 2:411-415

Chouinard G, Jones B, Remington G, Bloom D, Addington D, Mac Ewan GW, Labelle, Beauclair L, Amott W (1993): A Canadian multicenter placebo-controlled study of fixed doses of risperidone and haloperidol in the treatment of chronic schizophrenic patients. J Clin Psychopharmacol 13:25-40

Clark NL, Ray TS, Ragland RD (1963): Chlorpromazine in chronic schizophrenic women: rate of onset and rate of dissipation of drug effects. Psychosom Med 25:212-217

Coryell WH, Kelly MW, Perry PJ, Miller DD (1990): Haloperidol plasma levels and acute clinical change in schizophrenia. J Clin Psychopharmacol 10:397-402

Crow TJ(1980): Molecular pathology of schizophrenia; more than one disease process? BMJ 280:66-68

Davidson M, Kahn R, Powchik P, Warne P, Losonczy M, Kaminsky R, Apter S, Jaff S, Davis K (1991): Changes 
in plasma homovanillic acid concentrations in schizophrenic patients following neuroleptic discontinuation. Arch Gen Psychiatry 48:73-76

Dencker S, Malm U, Lepp M (1986): Schizophrenic relapse after drug withdrawal is predictable. ActaPsychiatr Scand 73:181-185

Dixon L, Gunvant T, Conley R, Ross D, Cascella N, Tamminga C (1993): Changes in psychopathology and dyskinesia after neuroleptic withdrawal in a double-blind design. Schizophrenia Res 10:267-271

Ekstrom D, Quade D, Golden RN (1990): Statistical analysis of repeated measures in psychiatric research. Arch Gen Psychiatry 47:770-772

Endicott J, Spitzer RL, Fleiss JL, Cogen J (1976): The Global Assessment Scale: A procedure for measuring overall severity of psychiatric disturbances. Arch Gen Psychiatry 33:766-771

Folstein MF, Folstein SE, McHugh P (1975): 'Mini-Mental State': A practical method for grading the cognitive state of patients for the clinician. J Psychiatr Res 12:189-198

Gittelman-Klein R, Klein DF (1969): Premorbid asocial adjustment and prognosis in schizophrenia. J Psychiatr Res 7:35-53

Glazer W, Bowers M, Charney D, Heninger G (1989): The effect of neuroleptic discontinuation on psychopathology, involuntary movements, and biochemical measures in patients with persistent tardive dyskinesa. Biol Psychiatry 26:224-233

Goldberg SC (1985): Negative and deficit symptoms in schizophrenia do respond to neuroleptics. Schizophr Bull 11:453-456

Goldman RS, Tandon R, Liberzon I, Greden JF (1992): Measurement of depression and negative symptoms in schizophrenia. Psychopathology 25:49-56

Green A, Faraone S, Brown W (1990): Prolactin shifts after neuroleptic withdrawal. Psychiatry Res 32:213-219

Gur RE, Mozley PD, Resnick SM, Levick S, Erwin R, Saykin AJ, Gur RC (1991): Relations among clinical scales in schizophrenia. Am J Psychiatry 148:472-478

Guy W, Cleary P, Bonato RR: Methodological implications of a large central data system. Proceedings of IXth Congress, CINP, Exerpta Medica, Amsterdam, 1975

Hamilton M (1960): A rating scale for depression. J Neurol Neurosurg Psychiatry 23:56-62

Heinrichs D, Carpenter W (1985): Experience with a drugfree month in schizophrenic patients. Psychopharmacol Bull 21:117-119

Hogarty G, Ulrich R, Mussare F, Aristigueta N (1976): Drug discontinuation among long term, successfully maintained schizophrenic outpatients. Diseases of the Nervous System (September), pp. 494-500

Jackson JH (1875): On temporary mental disorders after epileptic paroxysms. West Riding Lunatic Asylum Medical Report 5:105-129

Johnstone EC, Crow TJ, Frith CD, Carney MWP, Price JS (1979): Mechanism of the antipsychotic effect in the treatment of acute schizophrenia. Lancet 1:848-851

Kane J, Honigfeld G, Singer J, Meltzer HY (1988): Clozapine for the treatment-resistant schizophrenic. A double-blind comparison with chlorpromazine. Arch Gen Psychiatry 45:789-796

Kirkpatrick B, Buchanan RW, Maeda K, Carpenter WT, Jauch D, Tamminga CA (1989a): Effect of neuroleptic withdrawal on plasma prolactin: A possible marker of receptor adaptation. Biol Psychiatry 26:131-138

Kirkpatrick B, Buchanan RW, McKenney PD, Alphs LD, Carpenter WT (1989b): The schedule for the deficit syndrome: An instrument for research in schizophrenia. Psychiatry Res 30:119-124

Kraepelin E, Barclay RM, Robertson GM (1919): Dementia Praecox and Paraphrenia (translated). Edinburgh, E\& S Livingston

Liddle PF (1987): The symptoms of chronic schizophrenia: a reexamination of the positive-negative dichotomy. $\mathrm{Br}$ J Psychiatry 151:145-151

Lieberman J, Kane J, Sarantakos S (1987): Prediction of relapse in schizophrenia. Arch Gen Psychiatry 44:597-603

Marder S, van Kammen D, Bunney Jr W (1979): Prediction of drug-free improvement in schizophrenic psychosis. Arch Gen Psychiatry 36:1080-1085

Mauchly JW (1940): Significance tests for sphericity of a normal n-variate distribution. Annals of Mathematical Statistics 11:204-209

Meltzer HY (1991): Pharmacological Treatment of Negative Symptoms. In Greden JF, Tandon R (eds), Negative Schizophrenic Symptoms: Pathophysiology and Clinical Implications. Washington, DC; American Psychiatric Press, Inc.

Meltzer HY, Sommers AA, Luchins DJ (1986): The effect of neuroleptics and other psychotropic drugs on negative symptoms in schizophrenia. J Clin Psychopharmacol 6:329-338

Miller DD, Arndt SV, Andreasen NC (1993): Alogia, attentional impairment, and inappropriate affect: Their status in the dimensions of schizophrenia. Comp Psychiatry 34:221-226

Miller DD, Perry PJ, Cadoret RJ, Andreasen NC (1994): The effect of clozapine on negative symptoms in treatmentresistant schizophrenics. Comp Psychiatry 35(1): 1-6

Naber D, Albus M, Bürke H, Müller-Spahn F, Münch U, Reinertshofer T, Wissmann J, Ackenheil M (1985): Neuroleptic withdrawal in chronic schizophrenia: Clinical and endocrine variables relating to psychopathology. Psychiatry Res 16:207-219

Perenyi A, Frecska E, Bagdy G, Revai K (1985): Changes in mental condition, hyperkinesias, and biochemical parameters after withdrawal of chronic neuroleptic treatment. Acta Psychiatr Scand 72:430-435

Prien R, Cole J, Belkin N (1968): Relapse in chronic schizophrenics following abrupt withdrawal of tranquilizing medication. Br J Psychiatry 115:679-686

Rogan JC, Keselman HJ, Mendoza JL (1979): Analysis of repeated measures. Br J of Math Stat Psychol 32:269-286

Serafatinides EA, Collins S, Clark MI (1972): Haloperidol, clopenthixol, and chlorpromazine in chronic schizophrenia. J Nerv Ment Dis 154:31-42

Simpson GM, Angus JS (1970): A rating scale for extrapyramidal side effects. Acta Psychiatr Scand. 212 (suppl):11-19 
Siris SG (1991): Diagnosis of secondary depression in schizophrenia. Schizophr Bull 17:75-98

Strauss JS, Carpender WT, BarkoJJ(1974): The diagnosis and understanding of schizophrenia, part 3: Speculation on the process that underlies schizophrenic symptoms and signs. Schizophr Bull 3:240-244

Tandon R, Goldman RS, Goodson J, Greden JF (1990): Mutability and relationship between positive and negative symptoms during neuroleptic treatment in schizophrenia. Biol Psychiatry 27:1323-1326

van Kammen D, Peters J, van Kammen W, Nugent A, Goetz
K, Yao J, Linnoila M (1989): CSF Norepinephrine in schizophrenia is elevated prior to relapse after haloperidol withdrawal. Biol Psychiatry 26:176-188

Weinberger D, Llewellyn B, Klein S, Wyatt R (1981): Drug withdrawal in chronic schizophrenic patients: In search of neuroleptic-induced supersensitivity psychosis. JClin Psychopharmacol 1:120-123

Zander K, Fischer B, Zimmer R, Ackenheil M (1981): Long term neuroleptic treatment of chronic schizophrenic patients: Clinical and biochemical effects of withdrawal. Psychopharmacology 73:43-47 